\title{
On-bead analysis of substrate specificity of caspases using peptide modified by quaternary ammonium group as ionization enhancers
}

\author{
Remigiusz Bąchor ${ }^{1}$, Aneta Paluch ${ }^{1}$, Wioletta Rut ${ }^{2}$, Marcin Drag ${ }^{1}$, Zbigniew Sze wczuk $^{1}$ \\ ${ }^{1}$ Faculty of Chemistry, Wrocław University, Poland \\ ${ }^{2}$ Departmentof Bioorganic Chemistry, Faculty of Chemistry, Wroclaw University of Technology, Poland
}

https://doi.org/10.17952/35EPS.2018.212

Caspases are proteolytic enzymes at the heart of networks that govern apoptosis and inflammation. Therefore the investigation of new substrates for monitoring of their activity is of great importance. The one-beadone-compound (OBOC) peptide libraries, allow for obtaining and screening a wide range of compounds in short time, are commonly used in such kind of investigation and electrospray mass spectrometry (ESI-MS) is currently the method of choice for the identification of compounds on single beads. However, up to now, the analysis of on-bead peptide proteolysis and cleavage site have not been investigated by MS due to the necessity of analysis of trace amount of compound obtained from a single resin bead (femtomole level, $10^{-15}$ mole) which may be insufficient for reliable sequence analysis. Previously we invented the application of quaternary ammonium (QA) group as ionization tags for ultrasensitive sequencing of peptide obtained from a single resin beads of OBOC peptide library by tandem mass spectrometry $[1,2]$.

The aim of this work was to analyze the on-bead peptide proteolysis by using resin-bound peptide modified by ionization tag and LC-ESI-MS/MS technique (Fig. 1). Ionization tag in the form of quaternary ammonium(QA) group (4-aza-1-azoniabicyclo[2.2.2] octylammonium acetyl group), increasing the ionization efficiency during ESI-MS analysis, was located at the N-terminus of the synthesized peptides. The on-resin peptide bond cleavage by caspase, occurring even with low yield, results in the formation of QA-peptide present in supernatant which may be analyzed by ESI-MS.

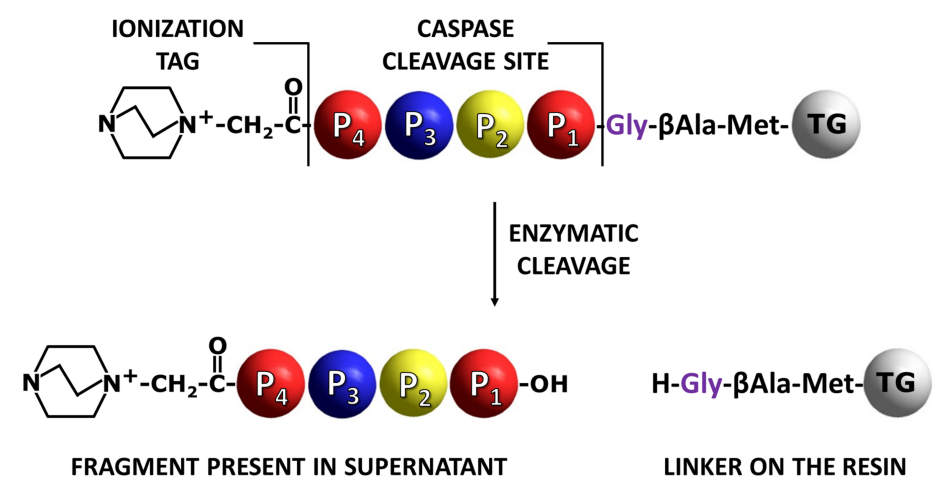

Figure 1: Schematic presentation of the idea of on-bead peptide proteolysis analysis using resin-bound peptide modified by ionization tag.

Model peptide sequences bearing QA group at the N-terminus were synthesized on the TentaGel $\mathrm{HL}-\mathrm{NH}_{2}$ resin where the peptide substrates were connected to the resin by the linker containing methionine residue, allowing peptide cleavage by $\mathrm{CNBr}, \beta$ Ala as a spacer and Gly as the best residue for caspases at $\mathrm{P}_{1}$ ' position (Fig. 1). To obtain high proteolytic activity the $\mathrm{DEVD} / \mathrm{G}$ sequence was selected as the optimal motif for the executioner caspases, caspase 3 and 7 (Fig. 2). It was also presented that the caspase 3 and 7 cleave the DEVE/G sequence however very poorly [3]. Therefore this motif was used to analyze the sensitivity of the proposed here analytical tool for the on-bead monitoring of caspase activity. The DEVA/G sequence was synthesized as a control due to its stability towards caspases. 

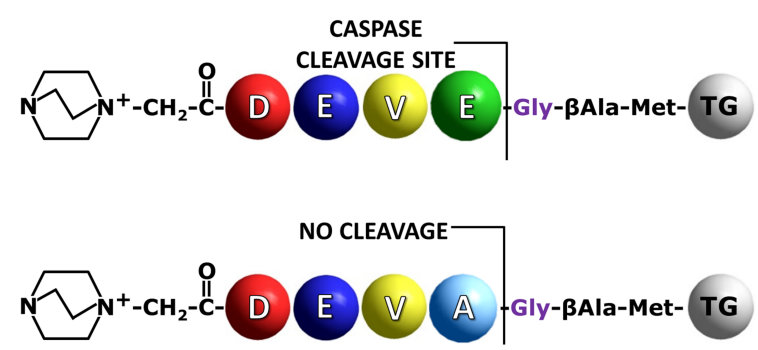

Figure 2: Schematic presentation of the synthesized model sequences containing QA group for the on-bead analysis of peptide bond hydrolysis by caspases.

After the peptide synthesis the fixed charge tag in the form of 4-aza-1-azoniabicyclo[2.2.2] octylammonium acetyl group was synthesized at the $\alpha$-amino group of the $\mathrm{N}$-terminal amino acid residue according to the procedure described by us previously [4]. Then the side chain protecting groups were removed under acidic conditions and $3 \mathrm{mg}$ of the obtained QA-modified peptidyl resins were incubated with a caspases 3 and 7 for 5 , $15,30,60$ and 180 minutes. The enzymatic reaction was terminated by the addition of formic acid $(5 \mu \mathrm{l})$ and the presence of fixed charge tagged peptides released to the supernatant after on-bead enzymatic digestion was analyzed by LC-ESI-MS/MS. The following transitions were investigated: for QA-DEVD-OH: $629.4 \rightarrow 268.1$ (b

A)

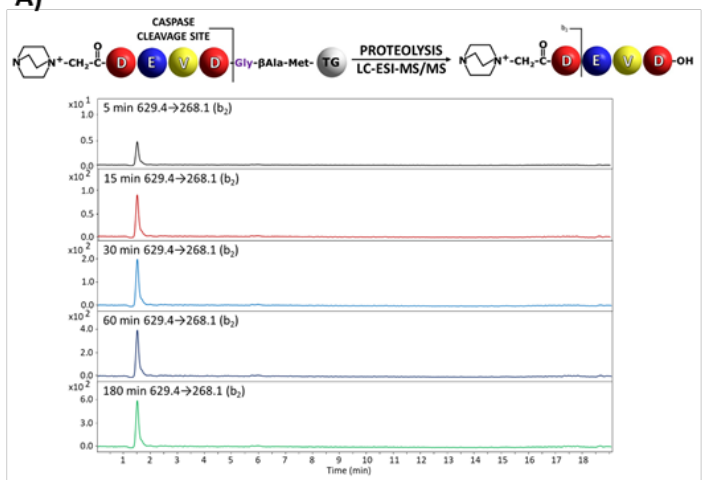

B)

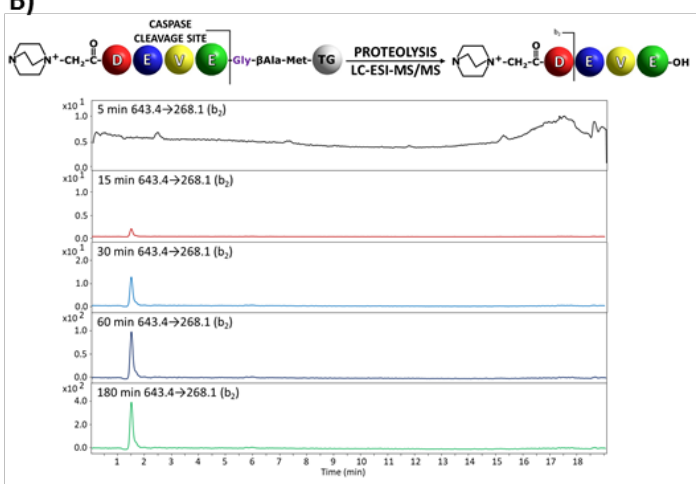

Figure 3: A) Chromatograms for QA-DEVD-OH peptide, analyzed transition $629.4 \rightarrow 268.1$ and for B) $Q A$ DEVE-OH peptide (analyzed transition $643.4 \rightarrow 268.1$ ) investigated in supernatant after incubation with caspase 3.

It was found that in the case of QA-DEVD/G sequence located on the resin the proteolysis product was present in the supernatant even after 5 minutes of enzymatic reaction. The hydrolysis of QA-DEVE/G sequence was also confirmed using our method however the proteolysis product was found in supernatant after 15 minutes of incubation with caspase. It is commonly known that the DEVE/G sequence is poorly recognized by caspases 3 and 7. Therefore the amount of compound released to the supernatant may be very small however the presence of QA tag increases the ionization efficiency of peptide allowing for the ultrasensitive analysis of compounds in complex mixture (supernatant). The signal corresponding to the hydrolysis of QA-DEVA/G sequence was not observed which was expected. The proposed method confirmed the known specificity of caspase 3 and 7 - cleavage after Asp residue, poor cleavage after Glu residue and no cleavage after Ala residue. The solution presented here is a new, rapid and straightforward method of the on-bead caspases activity analysis by LC-ESI-MS/MS technique. The application of fixed charge tag in the form of QA group allows the ultrasensitive analysis of peptide released even from a single resin bead. The proposed method may be used as a tool for the analysis of substrate specificity of enzymes.

This work was supported by a grant No. UMO-2015/17/D/ST5/01329 from the National Science Centre, Poland. 


\section{References}

[1] R. Bąchor, M. Cydzik, M. Rudowska, A. Kluczyk, P. Stefanowicz, Z. Szewczuk. Mol. Divers., 16, 613 (2012).

[2] R. Bąchor, A. Kluczyk, P. Stefanowicz, Z. Szewczuk. Mol. Divers., 17, 605 (2013).

[3] M. Poręba, A. Szalek, W. Rut, P. Kasperkiewicz, I. Rutkowska-Wlodarczyk, S.J. Snipas, Y. Itoh, D. Turk, B. Turk, Ch.M. Overall, L. Kaczmarek, G.S. Salv esen, M. Drag. Sci. Rep., 23, 7, 43135 (2017).

[4] M. Cydzik, M. Rudowska, P. Stefanowicz, Z. Szewczuk. J. Pept. Sci., 17, 445 (2011). 